\title{
Leucismo en el coatí de nariz blanca Nasua narica (Mammalia: Carnivora), en Quintana Roo, México
}

\author{
Adrián Silva-Caballero ${ }^{1 *}$, Fernando Montiel-Reyes ${ }^{1}$, Eduardo Sánchez-Garibay ${ }^{1}$ y Jorge Ortega ${ }^{1}$.
}

Introduction: Chromatic aberrations happen infrequently in mammals, usually as a result of the expression of a recessive gene. Leucism is the partial or total loss of pigmentation; this condition is the result of genetic mutations. This manuscript is the first report of leucistic individuals of White-nosed coati Nasua narica in Mexico.

Methods: In September-October 2012, in the course of a faunal survey in the Yucatán Peninsula two atypical White-nosed coatis Nasua narica (Linnaeus 1766) was sighted and photographed in two different localities: "Crococun" regional zoological park, in Puerto Morelos, Quintana Roo, México $\left(20^{\circ} 52.822^{\prime} \mathrm{N}\right.$ y $-86^{\circ} 53.222^{\prime} \mathrm{W}$, $8 \mathrm{~m})$, and "Condominio Club Real, Playacar Fase 1", in Playa del Carmen, Quintana Roo, México (20³6.775' N y $\left.-87^{\circ} 05.711^{\prime} \mathrm{W}, 10 \mathrm{~m}\right)$.

Results: The animals, a juvenile and an adult, both females, showed a lack of pigmentation in the back, belly and members, coloration ranged from white to creamy-white; both cases had a slightly brown color in the neck and rings of the same color in the tail (Figura 1). Such pigmentation pattern is similar to the reported for leucistic individuals (Buckley 1982).

Discussion and Conclusions: Little is known about the cost to an individual under those conditions associated with this reduction in pigmentation; some authors consider leucism as a disadvantage, as it can be a determining factor for survival, because this condition increases visibility to potential predators and therefore suffer greater selection pressure (Chętnicki et al. 2007). The interpretation of the biological significance of atypical patterns of coloration still remains uncertain; however, it is known that the leucism can provide indirect evidence of the underlying genetic variability, genetic change to variation in fitness-related traits and/or population structure (Acevedo and Aguayo 2008). Therefore, it is necessary to perform detailed genetic studies to generate knowledge about the cause of the abnormalities in the color of the fur and to clarify whether further environmental or biological factors are involved.

Key words: genetic mutation, mammals, mesocarnivores, Mexico, pigmentation.

\section{Resumen}

Las aberraciones cromáticas ocurren en mamíferos con poca frecuencia. El leucismo es la pérdida parcial o total de la pigmentación, dicha condición deriva de mutaciones genéticas. En este trabajo se presenta el primer registro de individuos leucísticos del coatí de nariz blanca Nasua narica en México.

Palabras clave: mamíferos, mesocarnívoros, mutación genética, México, pigmentación.

'Laboratorio de Bioconservación y Manejo, Departamento de Zoología, Posgrado en Ciencias Quimicobiológicas, Escuela Nacional de Ciencias Biológicas, Instituto Politécnico Nacional. Prolongación de Carpio y Plan de Ayala s/n, México, Distrito Federal 11340, México. E-mail: Isilvac@ipn.mx (AS-C), fercho_mom@hotmail.com (FM-R), weropsky_999@hotmail.com (ESG), artibeus2@aol.com (JO)

${ }^{*}$ Corresponding author 
En los organismos llegan a ocurrir variaciones en su coloración natural, dichas aberraciones cromáticas generalmente se deben a la expresión de un gen recesivo (Owen y Shimmings 1992). Es probable que estos patrones de coloración alterados podrían estar generados por factores externos, tales como la dieta (Buckley 1982), el hábitat (Peles et al. 1995) el aislamiento poblacional por la fragmentación del hábitat (Holyoak 1978), la contaminación radioactiva (Moller y Mousseau 2001), las enfermedades o lesiones en los folículos celulares (Balderas y Campos 2011). Estas expresiones de genes mutantes (Bensch et al. 2000), generalmente se encuentran relacionados con la falta de producción de tirosinasa, enzima que está involucrada en la síntesis de melanina, responsable de dar la coloración a la piel o pelo (Balderas y Campos 2011; Guevara et al. 2011; García-Morales et al. 2012).

Según las características fenotípicas de los individuos, los déficits de coloración se pueden clasificar en cuatro tipos: albinismo, que es la pérdida total de los pigmentos en todo el cuerpo. Dilución, cuando la tonalidad del color se reduce al igual que los pigmentos. Esquizocroísmo, en donde un pigmento no se expresa sin afectar a otro. Leucismo, condición que se refiere a la reducción de la pigmentación parcial o total, sin afectar las partes blandas (Buckley 1982). Los animales que presentan leucismo por lo general tienen el pelo o piel de color blanco, pero los ojos y uñas mantienen su coloración normal (Jehl 1985; Acevedo y Aguayo 2008; Guevara et al. 2011; García-Morales et al. 2012). El leucismo se ha registrado en varios grupos animales, en mamíferos se tienen registros en cánidos (López-González 2011), erinaceomorfos (Morris y Tutt 1996), mustélidos (Tortato y Althoff 2007), pinnípedos (Acevedo y Aguayo 2008), quirópteros (García-Morales et al. 2012), sorícidos (Guevara et al. 2011) y úrsidos (Ritland et al. 2001).

Nasua narica es considerado como el único carnívoro verdaderamente sociable que habita los bosques neotropicales (Valenzuela 1998). Su distribución abarca desde el sur de Estados Unidos hasta Colombia, Ecuador y Perú, al oeste de los Andes; en México en todo el país excepto en la Península de Baja California y el Altiplano Central (Valenzuela 1998; Aranda 2012). Habita todos los tipos de bosque tropical, incluyendo el manglar, bosque mesófilo de montaña, bosques mixtos de coníferas y encinos, incluso matorral xerófilo (Aranda 2012). Es un mamífero de tamaño mediano de color generalmente pardo en el dorso, con diferentes tonalidades desde grisáceo claro a un rojizo amarillento, llegando a tener manchas claras en la nuca y alrededor de los ojos. Puede llegar a presentar anillos poco contrastantes en la cola (Gompper 1995; Aranda 2012).

En la Península de Yucatán entre Septiembre-Octubre 2012, se registraron mediante observación directa, dos ejemplares con coloración propia de individuos leucísticos. El primero en el parque zoológico regional "Crococun", ubicado en Puerto Morelos, municipio Benito Juárez, Quintana Roo, México (2052. 822' N y -8653.222' W, 8 m). El ejemplar correspondía a una hembra juvenil, la cual se encontraba bajo resguardo en dicho zoológico por indicaciones de la Procuraduría Federal de Protección al Ambiente (PROFEPA). Su procedencia fue desconocida, sólo se sabía que era un animal incautado, procedente de alguna parte de la península de Yucatán. Las medidas del ejemplar fueron $611 \mathrm{~mm}$ de longitud total, $292 \mathrm{~mm}$ de cola vertebral, $55 \mathrm{~mm}$ de la pata trasera derecha, $23 \mathrm{~mm}$ de oreja, con $1.1 \mathrm{~kg}$ de peso.

El segundo, fue un avistamiento dentro de las instalaciones de un complejo turísticoresidencial conocido como "Condominio Club Real, Playacar Fase 1", en Playa del Carmen, 
Municipio de Solidaridad, Quintana Roo, México $\left(20^{\circ} 36.775^{\prime} \mathrm{N}\right.$ y $\left.-87^{\circ} 05.711^{\prime} \mathrm{W}, 10 \mathrm{~m}\right)$. El ejemplar era una hembra adulta perteneciente a una banda de aproximadamente 30 individuos, dicho grupo habita entre los relictos de selva que aún quedan entre las villas, diversos hoteles y un campo de golf (Figura 1). Ambos individuos mostraban una falta de pigmentación en el dorso, vientre y miembros, la coloración variaba entre blanco y blancocremoso, con la base del pelo blanco y las puntas en color crema; ambos casos presentaban una coloración ligeramente café en la nuca así como anillos del mismo color en la cola. Poco se sabe sobre el costo para un individuo en esas condiciones asociado con esta reducción de la pigmentación; algunos autores consideran al leucismo como una desventaja, ya que puede llegar a ser un factor determinante en cuanto a la supervivencia, debido a que dicha condición aumenta la visibilidad ante posibles depredadores y, por consiguiente, sufrir una mayor presión de selección (Chętnicki et al. 2007; Jogahara et al. 2008; Balderas y Campos 2011).

Figura 1. Individuo de Nasua narica con leucismo junto con otros individuos de su banda los cuales presentan coloración normal.

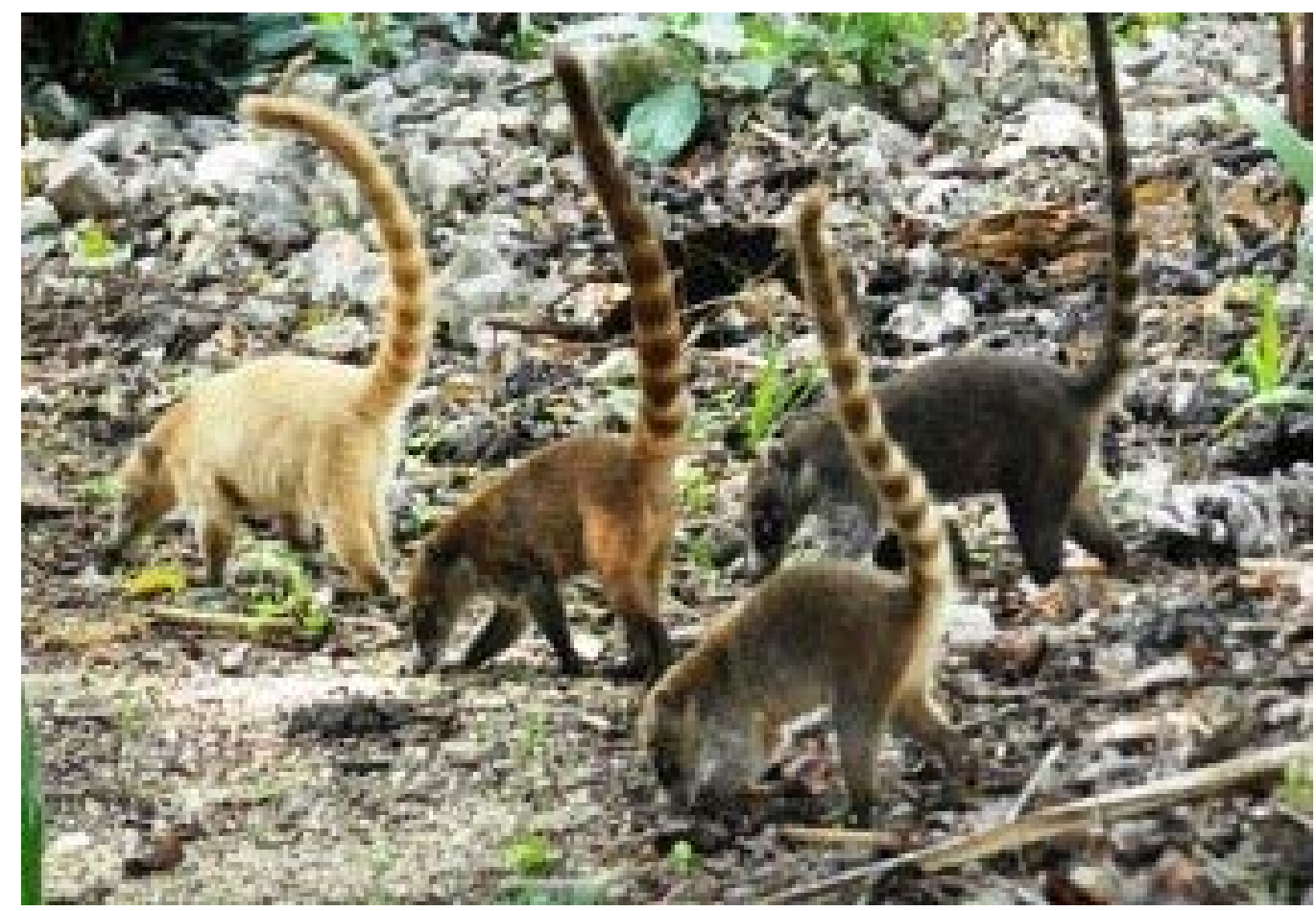

Por otra parte, se cree que esta aberración cromática reduce la viabilidad debido a factores patológicos tales como defectos sensoriales o nerviosos, anemia, baja fertilidad, aumento de la susceptibilidad a enfermedades y falta de visión (Jehl 1985; Acevedo y Aguayo 2008). A pesar de esto, existe el registro de algunos individuos que han alcanzado la mayoría de edad y por tanto, la etapa reproductiva (Acevedo y Aguayo 2008). Aparentemente, la selección natural ha producido una baja frecuencia del alelo que produce el leucismo el cual probablemente pueda reducir la condición física en la vida silvestre; de lo contrario el alelo podría ser frecuente (Chętnicki et al. 2007; Guevara et al. 2011; Rodríguez-Pinilla y Gómez-Martínez 2011). Las aberraciones cromáticas se observan frecuentemente en especies gregarias o sedentarias y se piensa que podrían ser más comunes en poblaciones pequeñas y aisladas (Bensch et al. 2000; Chętnicki et al. 2007), debido a que la endogamia aumenta la probabilidad de que los alelos recesivos se expresen (Bensch et al. 2000; Balderas 
y Campos 2011). La interpretación de la importancia biológica de los patrones atípicos de coloración es aún incierta. Sin embargo, se sabe que el leucismo puede proporcionar evidencia indirecta de la variabilidad genética subyacente (Jehl 1985), así como el cambio genético para las variaciones en cuanto a los atributos relacionados con la adecuación y la estructura de las poblaciones (Acevedo y Aguayo 2008). Por lo anterior, es necesario efectuar estudios genéticos detallados para generar conocimiento sobre la causa de las anormalidades en la coloración del pelaje y poder esclarecer si hay factores ambientales o biológicos interviniendo adicionalmente (Jogahara et al. 2008; Guevara et al. 2011).

Agradecemos a E. M. Río Aguilar, Director del parque zoológico regional "Crococun", así como a J. Carballar Osorio y A. Verastegui por las facilidades otorgadas dentro de sus instalaciones; de la misma manera agradecemos el apoyo invaluable de D. Chavira Martínez en Playa del Carmen, así como por otorgarnos la autorización del uso de sus fotografías para documentar este escrito. También agradecemos al Cleveland Metroparks Zoo por el otorgamiento del Scott Neotropical Fund para la realización de este proyecto.

Acevedo, J., y M. Aguayo. 2008. Leucistic South American sea lion in Chile, with a review of

Literatura citada anomalously color in otariids. Revista de Biología Marina y Oceanografía 43:413-417.

Aranda, M. 2012. Manual para el rastreo de mamíferos de México. CONABIO. Ciudad de México, México.

Balderas, A. M. J., y G. R. Campos. 2011. Primer informe de leucismo en la paloma de collar Streptocelia decaocto (Columbiformes), especie exótica de México. Cuadernos de Investigación UNED. Edición en Línea 31:85-88.

Bensch, S., B. Hansson, D. Hasselquist, y B. Nielsen. 2000. Partial albinism in a semi-isolated population of great reed warblers. Hereditas 133:167-170.

Buckley, P. A. 1982. Avian genetic. Pp. 21-110 en Diseases of cage and aviary birds (Petrak, M., ed.). Segunda Edición. Lea and Febiger Press. Philadelphia, EE.UU.

Chętnicki, W., S. Fedyk, y U. Bajkowska. 2007. Cases of coat colour anomalies in the common shrew, Sorex araneus L. Folia Biológica (Kraków) 55:73-76.

García-Morales, R., D. T. Duran, E. S. A. Gómez, C. E. Moreno, y M. S. Akmentis. 2012. Registro de leucismo en Sturnira ludovici y Artibeus jamaicensis (Phyllostomidae) en México. Chiroptera Neotropical 18:1101-1105.

GompPeR, M. E. 1995. Nasua narica. Mammalian Species 487:1-10.

Guevara, L., H. E. Ramírez-Chaves, y F. A. Cervantes. 2011. Leucismo en la musaraña de orejas cortas Cryptotis mexicana (Mammalia: Soricomorpha), endémica de México. Revista Mexicana de Biodiversidad 82:731-733.

Holyoak, D. T. 1978. Variable albinism of the flight feathers as an adaptation of recognition of individual birds in some Polynesian populations of Acrocephalus warblers. Ardea 66:112-117.

JeHL, J. 1985. Leucism in pared grebes in western North America. The Condor 87:439-441.

Jogahara, T., G. Ogura, G. Higa, O. Ishibashi, y S. Oda. 2008. Survey and capture of albinolike house musk shrews (Suncus murinus) in Okinawa, Japan, and a preliminary report regarding inheritance of the albino-like mutation. Mammal Study 33:121-124. 
López-González, C. A. 2011. Registro de un coyote (Canis latrans) blanco en el Desierto Chihuahuense de Durango, México. Acta Zoológica Mexicana 27:871-873.

Moller, A. P., y T. A. Mousseau. 2001. Albinism and phenotype of barn swallows. Evolution 55:2097-2104.

Morris, P., Y A. Tutr. 1996. Leucistic hedgehogs on the island of Alderney. Journal of Zoology 239:387-389.

Owen, M., y P. Shimmings. 1992. The occurrence and performance of leucistic barnacle geese, Branta leucopsis. Ibis 134: 22-26.

Peles, J. D., M. F. Lucas, y G. W. Barrett. 1995. Population dynamics of agouti and albino meadow voles in high-quality, grassland habitats. Journal of Mammalogy 76:10131019.

Ritland, K., C. Newton, y H. D. Marshal. 2001. Inheritance and population structure of the white-phased "Kermode" black bear. Current Biology 11:1468-1472.

Rodríguez-Pinilla, Q., y M. J. Gómez-Martínez. 2011. Leucismo incompleto en Turdus fuscater (Passeriformes: Turdidae) en los Andes Colombianos. Boletín Científico Centro de Museos, Museo Nacional de Historia Natural 15:63-67.

ToRTATo, F. R., Y S. L. Althoff. 2007. Variações na coloração de iraras (Eira barbara Linnaeus, 1758 - Carnivora, Mustelidae) da Reserva Biológica Estadual do Sassafrás, Santa Catarina, sul do Brasil. Biota Neotropica 7:365-367.

Valenzuela, D. 1998. Natural history of the white-nosed coati, Nasua narica, in a tropical dry forest of western of México. Revista Mexicana de Mastozoología 3:26-44.

Sometido: 30 de enero de 2014

Revisado: 11 de septiembre de 2014

Aceptado: 17 de septiembre 2014

Editor asociado: Juan Pablo Gallo 
LEUCISMO EN NASUA NARICA

844 THERYA Vol.5(3): 839-843 